\title{
Application of total artificial heart in patients with primary malignant cardiac tumors-current treatment strategies
}

\author{
Jiewen Xiao, Nicolas Brozzi, Renzo Cifuentes, Ali Ghodsizad, Matthias Loebe \\ Division of Thoracic Transplantation \& Mechanical Circulatory Support, Department of Surgery, Miller School of Medicine, University of Miami, \\ Coral Gables, FL, USA \\ Correspondence to: Matthias Loebe, MD, PhD. Professor of Surgery, Division Chief, Division of Thoracic Transplantation \& Mechanical Circulatory \\ Support, Department of Surgery, Miller School of Medicine, University of Miami, Coral Gables, FL 33146, USA. Email: mxl1039@med.miami.edu.
}

Submitted Jan 20, 2020. Accepted for publication Feb 11, 2020.

doi: 10.21037 /acs.2020.02.12

View this article at: http://dx.doi.org/10.21037/acs.2020.02.12

Primary tumors of the heart and great vessels are extremely rare, with the estimated prevalence of $0.0017 \%$ to $0.3 \%$ (1). Malignant tumors represent only $15-25 \%$ of primary cardiac tumors $(2,3)$. Because of this, most clinicians may experience only a few cases in their lifetime, resulting in the lack of core knowledge of the incidence, histological characteristics, clinical manifestations, pathogenesis and the effective treatment strategy of malignant cardiac tumors. Primary malignant cardiac tumors can present at any age with a peak incidence in the $4^{\text {th }}$ and $5^{\text {th }}$ decade of life (4). Female patients are slightly more frequently affected $(3,4)$. Sarcomas are the most common primary malignant cardiac tumors, followed by lymphomas and other types of malignant tumors (4). Angiosarcomas are the most common type of sarcoma, followed by leiomyosarcomas and rhabdomyosarcomas (5).

The clinical manifestations of malignant cardiac tumors are nonspecific and vary from asymptomatic cardiac imaging findings to shock or sudden cardiac death. Symptoms depend on the cardiac structures involved and the biological characteristics of the tumor (6). Symptoms can be categorized in disturbance of hemodynamics, distal embolization, and constitutional symptoms. The size, site and growing pattern of the tumor determine the severity of hemodynamics compromise. If one of the valves and outflow tract are involved, congestive heart failure or syncope may occur. Distal embolization from the left atrium or ventricle may result in stroke, myocardial infarction, acute limb ischemia and/or abdominal organ infarction, while a mass in the right heart may result in pulmonary embolism.

With more and more experience being collected, there is consensus that radical resection of the tumor seems to be the best treatment option even though tumor recurrence cannot be totally avoided. Negative surgical margins status are important for survival after resection of the tumor.

\section{Auto-transplant}

It involves cardiac excision, ex-vivo tumor resection, cardiac reconstruction and reimplantation, providing the opportunity for resection of complex left-sided cardiac tumors. Benefits of this technique include improved accessibility and ability to perform radical tumor resection together with reliable cardiac reconstruction (7).

\section{Heart transplant}

Some of the tumors cannot be radically resected by conventional surgical procedures or auto-transplantation. The tumor size or the extent of infiltration can be relatively large in some patients (8). After resection of the tumor, it is impossible to reconstruct the heart's structure or maintain its function. In this situation, heart transplantation seems to be an alternative method. Transplant in this clinical setting brings an ethical concerns regarding utilization of scarce resource of cardiac donation for treatment of potentially recurrent malignancy.

\section{Total artificial heart}

Experience with the application of total artificial heart in malignant cardiac tumors is scarce due both to the rarity 
of the disease and low utilization rate of the total artificial heart in this kind of patients. In the published literature most of these patients recovered from surgery to some extent in the early postoperative period, but the rate of complications was high, mainly related to complications associated to postoperative anticoagulation, including mediastinal hematoma, pulmonary metastases, cerebral hemorrhage or liver failure, which determined an overall postoperative mortality of $57 \%(9,10)$. Anticoagulation is very important for patients with implantation of total artificial heart. Excess or deficiency of warfarin will result in hemorrhage or embolization respectively. The patients' malignant state is very different from those who do not have malignant tumors and may alter the coagulation function; specific anticoagulation protocols might be needed to decrease the rate of complications and improve postoperative outcomes. The role of chemotherapy or radiation therapy is controversial. Some studies found that adjuvant or neo-adjuvant therapy have no effect on the treatment, but others showed the combination of surgery and adjuvant therapy is effective. As a mechanical device, total artificial heart is "immune" to chemotherapy or radiation-related toxicity, facilitating the goal of complete systemic tumor remission. Before and during the adjuvant therapy, one should pay close attention to the vital organ function and extracardiac metastases.

Most patients who presented with either inflow/outflow tract obstruction or impaired ventricular function had significant progression of symptoms, leading to urgent surgery. The total artificial heart has the additional advantage of immediate availability at implanting centers, in contrast to the delays that would be required for extensive work up and waiting period for heart transplantation. The SynCardia total artificial heart is currently the only FDAapproved total artificial heart. Several dependent studies from large registries showed that pre- and post-transplant survival and outcomes of the patients with total artificial heart did not have significant difference from that of the patients with other biventricular assist devices. Despite this, there are indeed some differences. The SynCardia total artificial heart suits the patients who have larger body size; the size criteria is cardiothoracic ratio $>0.5$, posterior sternum to anterior vertebral column distance $>10 \mathrm{~cm}$ at $\mathrm{T} 10$, and body surface area $>1.7 \mathrm{~m}^{2}$. This body size limit restricts its application in smaller body patients such as women and adolescents. Since the SynCardia total artificial heart requires the presence of both native atria to achieve optimal implantation, patients with malignant cardiac tumors who need radical resection of atriums may not be candidates to this device. When reconstruction of the atrial structures becomes necessary, attention has to be given to the fact that the total artificial heart will exercise negative pressure. Therefore, reconstruction with rigid materials, reinforced with glues, may be needed to keep the inflow of the total artificial heart open. The primary goal of the resection of cardiac tumors is to obtain clean margins and therefore intraoperative frozen sections of resection margins is needed. Repair of the pulmonary artery beyond the bifurcation may be necessary. Under the premise of the similarity in survival and outcomes, the choice of the devices is dependent on overall cost, patient size, device-related complications and center experience. Another question yet to be answered is how long total artificial heart support can be extended, before the patient is considered a candidate for heart transplantation after tumor resection.

In conclusion, the SynCardia total artificial heart can represent an effective therapy for carefully selected patients presenting with malignant cardiac tumors, allowing total resection of the cardiac tumor, and facilitating adjuvant therapy. Careful anticoagulation management is critical to prevent development of postoperative complications.

\section{Acknowledgments}

None.

\section{Footnote}

Conflicts of Interest: The authors have no conflicts of interest to declare.

\section{References}

1. Hudzik B, Miszalski-Jamka K, Glowacki J, et al. Malignant tumors of the heart. Cancer Epidemiol 2015;39:665-72.

2. Shapiro LM. Cardiac tumours: diagnosis and management. Heart 2001;85:218-22.

3. Barreiro M, Renilla A, Jimenez JM, et al. Primary cardiac tumors: 32 years of experience from a Spanish tertiary surgical center. Cardiovasc Pathol 2013;22:424-7.

4. Oliveira GH, Al-Kindi SG, Hoimes C, et al. Characteristics and Survival of Malignant Cardiac Tumors: A 40-Year Analysis of \&gt;500 Patients. Circulation 2015;132:2395-402.

5. Burazor I, Aviel-Ronen S, Imazio M, et al. Primary malignancies of the heart and pericardium. Clin Cardiol 
2014;37:582-8.

6. Reich H, Czer L, Bannykh S, et al. Total Artificial Heart Bridge to Transplantation for a Patient With Occult Intracardiac Malignancy: Case Report. Transplant Proc 2015;47:2291-4.

7. Reardon MJ, Malaisrie SC, Walkes JC, et al. Cardiac autotransplantation for primary cardiac tumors. Ann Thorac Surg 2006;82:645-50.

8. Kremer J, Farag M, Arif R, et al. Total Artificial Heart

Cite this article as: Xiao J, Brozzi N, Cifuentes R, Ghodsizad A, Loebe M. Application of total artificial heart in patients with primary malignant cardiac tumors-current treatment strategies. Ann Cardiothorac Surg 2020;9(2):113-115. doi: 10.21037/acs.2020.02.12
Implantation After Undifferentiated High-Grade Sarcoma Excision. Med Sci Monit Basic Res 2016;22:128-31.

9. Bruckner BA, Rodriguez LE, Bunge R, et al. Large cardiac tumor managed with resection and two ventricular assist devices. Ann Thorac Surg 2014;97:321-4.

10. Bruckner BA, Abu Saleh WK, Al Jabbari O, et al. Total Artificial Heart Implantation after Excision of Right Ventricular Angiosarcoma. Tex Heart Inst J 2016;43:252-4. 